\title{
Estudio De Caso: Turismo Comunitario. Nivel De Satisfacción del visitante Sumak Kawsay, Provincia de Chimborazo - Ecuador
}

\author{
Jenry Ernesto López Romero \\ Rita Sulema Lara Vásconez \\ Manuel Antonio Abarca Zaquinaula \\ Luis Rafael Fiallos Ortega Norma \\ Norma Lissette Naranjo Sánchez \\ Rafael Marcos López Romero
}

Docentes investigadores Escuela de Ingeniería en Ecoturismo/

Escuela Superior Politécnica de Chimborazo, Ecuador

Doi:10.19044/esj.2018.v14n20p261 URL:http://dx.doi.org/10.19044/esj.2018.v14n20p261

\begin{abstract}
Ecuador is the country with the most widely diversified land mass per square mile in the whole world. It has countless live ecosystems and cultures, and it contains $11 \%$ of the world's biodiversity. These conditions have resulted to the development of the most interesting touristic activities. The province of Chimborazo is located at the very center of the Inter-Andean Highway and is known as the "Province of the Tall Peaks." The tallest of which is the volcano Mt. Chimborazo with a height of 6,310 mts (20,700 ft.). It, however, gave its name to the province itself. The train that runs down through the center of Ecuador to the Devil's Nose Mountain makes the province optimum for touristic activity. The small city of Calpi is one of the best known historic areas of Riobamba County. This is because in its interior, there are a lot of natural attractions for tourists. The community which is known as "The Royal Palace" tries to conserve its natural resources as well as to preserve its natural identity. During the past few years, this community has developed a lot of touristic activity since the Plan for Touristic Development is being organized for that zone. Tourism is being considered as an activity for the productive development that will augment and strengthen the local economy.
\end{abstract}

Keywords: Community tourism, sustainable development, mega-diverse, natural and cultural heritage, tourist attractions 


\section{Resumen}

Ecuador es el país más megadiverso del mundo por kilómetro cuadrado, contando con el $11 \%$ de la biodiversidad del mundo. Estas condiciones han permitido el desarrollo de las actividades turísticas en las diversas provincias y comunidades de dicho país, especialmente la Provincia de Chimborazo. Esta provincia se ubica en el centro del callejón interandino ecuatoriano y es conocida como la "provincia de las altas cumbres", siendo la más alta el volcán Chimborazo con una altura de $6.310 \mathrm{~m}$ (el cual le da el nombre a la provincia). De igual manera, el tren Ecuador con la ruta Nariz del Diablo, la parroquia Calpi en el cantón Riobamba con sus variados atractivos naturales y la comunidad de Palacio Real son la evidencia del desarrollo de la actividad turística durante los últimos años en la provincia antes mencionada, por lo que es necesaria una planificación para el manejo adecuado del turismo. Para ello se pretende formular el Plan Desarrollo Turístico Sostenible para la zona, en vista de que se ha considerado al turismo como una actividad de desarrollo productivo sostenible que permitirá dinamizar y fortalecer la economía local.

Palabras claves: Turismo comunitario, desarrollo sostenible, patrimonio natural y cultural, atractivos turísticos

\section{Introducción}

El turismo es considerado como un ente dinamizador y productivo a nivel mundial que ha evolucionado paulatinamente, de tal modo que en la actualidad constituye una de las actividades que generan mayores divisas para el mundo, contribuyendo así al mejoramiento de la calidad de vida de los habitantes. Para la Organización Mundial de Turismo (OMT) la erradicación de la pobreza es uno de los objetivos de desarrollo del milenio que se pretende contrarrestar a través del fomento del turismo sustentable mediante proyectos de desarrollo sostenible desde el punto de vista social, económico y medioambiental.

En el Ecuador el turismo es reconocido como uno de los sectores de mayor crecimiento, con una alta capacidad en la generación de empleos y divisas (SENPLADES, 2013). Por ello, el turismo es uno de los sectores priorizados para el cambio de la matriz productiva $(M C P E y C, 2014)$. A finales de la década de los 80 algunas comunidades en el Ecuador empezaron a incursionar en la actividad turística desarrollando una nueva propuesta alternativa como es el turismo comunitario (FEPTCE, 2010), considerado como un instrumento de lucha contra la pobreza y de generación de riqueza y oportunidades para todos (YAPU, 2006). El concepto de turismo comunitario se fundamenta en la creación de productos turísticos bajo el principio básico de la necesaria participación de la comunidad local. Esta forma de turismo se 
presenta como una oportunidad de establecer puentes entre la rentabilidad económica, la conservación del medio ambiente y el respeto socio cultural (Cruz Blasco, 2012), siendo considerada también como aquella actividad turística en donde el manejo, toma de decisiones y participación accionaria está en manos parcial o totalmente de las comunidades asentadas en un área natural apropiada para esta actividad (Prieto, 2011).

La actividad turística en el país durante las últimas décadas ha demostrado ser capaz de generar ingresos económicos, ofreciendo bienestar y prosperidad a varios sectores y constituyéndose en una política de Estado (MINTUR, 2016).

Por su parte, el turismo comunitario se ha presentado como un modelo de gestión que permite la relación de la comunidad con los visitantes desde una perspectiva intercultural en el desarrollo de viajes organizados con la participación consensuada de sus miembros, garantizando el manejo adecuado de los Recursos Naturales (FEPTCE, 2007).

En la parroquia Santiago de Calpi, a unos 15 kilómetros de la ciudad de Riobamba, capital de la provincia de Chimborazo, se encuentra un sector denominado Palacio Real, que forma parte de un plan de turismo en el que la comunidad viene trabajando desde el 2006 y que cuenta con algunos atractivos naturales y culturales.

Debido a la importancia de buscar las alternativas de desarrollo sostenible que contribuyan a mejorar la calidad de vida del sector rural, en la comunidad Palacio Real se desarrolló el producto turístico comunitario, que contribuye significativamente a mejorar el nivel de vida de los habitantes de las comunidades mediante la potencialización de sus recursos turísticos, creando una actividad complementaria a las formas productivas tradicionales.

El Centro Turístico Comunitario Sumak Kawsay ofrece varias actividades para realizar como: caminatas por los senderos para observar la flora y fauna nativa acompañados por una guía con vestuario típico de la comunidad junto con una llama, , visitas al museo cultural de la llama donde se puede conocer las características de los camélidos andinos y probar la gastronomía típica como la carne de llama (seco, fritada, lasaña y chuleta) acompañado con arroz de quinoa entre otros. Todos los productos del lugar se basan en la crianza y el aprovechamiento integral y sostenible de los camélidos andinos (llamas y alpacas): la población local aprovecha sus pieles para cubrirse del frío andino, sus carnes para alimentarse y su lana para confeccionar artesanías textiles y vestimentas.

La participación de las mujeres de la organización Nuevo Milenio ha tomado un papel de gran importancia puesto que en el Centro de Artesanías se exhiben y venden artículos elaborados de la fibra de la llama como: bufandas, ponchos, gorras, guantes, sacos e incluso ropa para bebé, entre otros. 
La visita a la comunidad es una gran experiencia por su belleza turística. Igualmente, la generosidad y amabilidad de sus pobladores lo harán sentir como en casa. Podrá también sentir el calor hogareño en el centro de hospedaje Quillapacari que ofrece todas las comodidades para que la estadía sea maravillosa e inolvidable.

El enfoque al cliente "focus costumer" se ha convertido en el gran protagonista de todas las empresas que deseen permanecer con éxito en el mercado.

La presente investigación tiene como objetivo determinar el nivel de satisfacción de los turistas nacionales e internacionales que visitan la comunidad. A través de un estudio de mercado se busca establecer las principales necesidades y requerimientos del visitante y su percepción con respecto al producto turístico. Brindar una impresión positiva, además de lograr la satisfacción del cliente, permitirá que los consumidores estén dispuestos a volver a consumir los servicios que presta la organización.

\section{Método}

Para el presente trabajo se emplearon métodos cuantitativos ya que permiten medir resultados. Se obtuvo información mediante la encuesta ya que otorga la posibilidad de aplicarla sobre muestras más amplias, las preguntas tabulables y no requieren demasiado tiempo.

\section{Análisis de la Demanda}

Se tomó como referencia los turistas que ingresaron al Centro de Turismo Comunitario Sumak Kawsay durante el período enero - marzo del año 2018. Durante este período ingresaron 104 turistas de los cuáles el 88\% son turistas internacionales y el restante son visitantes nacionales.

\section{Instrumento de Investigación}

El instrumento utilizado para recabar información fue la guía de encuesta que se encuentra en el anexo 1 para turistas nacionales y 2 aplicado a turistas internacionales.

\section{Técnica}

Utilizamos la investigación primaria. La técnica empleada fue la encuesta que permitió la recolección de información necesaria. 


\section{Resultados}

\section{Análisis de la Demanda}

Nivel de satisfacción del cliente internacional

\section{Alimentación}

\section{Preparación del platillo}

Cuadro 1. Percepción del turista internacional con respecto a la preparación del platillo

\begin{tabular}{|c|r|r|}
\hline Preparación del platillo & Frecuencia absoluta & \multicolumn{1}{|c|}{ Frecuencia relativa } \\
\hline Muy bueno & 10 & $18,2 \%$ \\
\hline Bueno & 12 & $22 \%$ \\
\hline Regular & 20 & $36,4 \%$ \\
\hline Malo & 13 & $23,6 \%$ \\
\hline
\end{tabular}

Análisis: El 36,4\% de los turistas consideran que la forma de preparación del platillo en cuanto a sabor, olor y color es regular.

\section{Calidad de los ingredientes}

Cuadro 2. Percepción del turista internacional con respecto a la calidad de los ingredientes

\begin{tabular}{|c|r|r|}
\hline Calidad de los ingredientes & \multicolumn{1}{|c|}{ Frecuencia absoluta } & \multicolumn{1}{c|}{ Frecuencia relativa } \\
\hline Muy bueno & 3 & $5 \%$ \\
\hline Bueno & 13 & $24 \%$ \\
\hline Regular & 29 & $53 \%$ \\
\hline Malo & 10 & $18 \%$ \\
\hline
\end{tabular}

Análisis: El 53\% de los clientes considera que la calidad de los ingredientes (frescos, orgánicos, naturales, de temporada) que se utilizan para elaborar los platillos es regular.

\section{Presentación del platillo}

Cuadro 3. Percepción del turista internacional con respecto a la presentación del platillo

\begin{tabular}{|c|r|r|}
\hline Presentación del platillo & Frecuencia absoluta & \multicolumn{2}{|c|}{ Frecuencia relativa } \\
\hline Muy bueno & 5 & $9 \%$ \\
\hline Bueno & 5 & $9 \%$ \\
\hline Regular & 29 & $53 \%$ \\
\hline Malo & 17 & $31 \%$ \\
\hline
\end{tabular}

Análisis: El 53\% de los visitantes considera que la presentación del platillo (montaje del plato, decoración del plato) es regular. 


\section{Forma de atención}

Cuadro 4. Percepción del turista internacional con respecto a la forma de atención

\begin{tabular}{|c|r|r|}
\hline Forma de atención & Frecuencia absoluta & Frecuencia relativa \\
\hline Muy bueno & 45 & $82 \%$ \\
\hline Bueno & 4 & $7 \%$ \\
\hline Regular & 4 & $7 \%$ \\
\hline Malo & 2 & $4 \%$ \\
\hline
\end{tabular}

Análisis: E1 82\% de los clientes considera que forma de atención en el servicio de alimentación es muy buena.

\section{Higiene del platillo}

Cuadro 5. Percepción del turista internacional con respecto a la higiene del platillo

\begin{tabular}{|c|r|r|}
\hline Higiene del platillo & Frecuencia absoluta & Frecuencia relativa \\
\hline Muy bueno & 48 & $87 \%$ \\
\hline Bueno & 5 & $9 \%$ \\
\hline Regular & 1 & $2 \%$ \\
\hline Malo & 1 & $2 \%$ \\
\hline
\end{tabular}

Análisis: El 87\% de los turistas considera que la higiene de los platillos es muy buena.

\section{Tiempo de espera}

Cuadro 6. Percepción del turista internacional con respecto al tiempo de espera

\begin{tabular}{|c|r|r|}
\hline Tiempo de espera & Frecuencia absoluta & \multicolumn{2}{|c|}{ Frecuencia relativa } \\
\hline Muy bueno & 5 & $9 \%$ \\
\hline Bueno & 3 & $5 \%$ \\
\hline Regular & 33 & $60 \%$ \\
\hline Malo & 14 & $25 \%$ \\
\hline
\end{tabular}

Análisis: El $60 \%$ de los visitantes considera que el tiempo de espera en el servicio de alimentación es regular ya que no se hace de una forma rápida y eficiente.

\section{Hospedaje}

\section{Calidad de la lencería}

Cuadro 7. Percepción del turista internacional con respecto a la calidad de la lencería

\begin{tabular}{|c|r|r|}
\hline Calidad de la lencería & Frecuencia absoluta & \multicolumn{1}{|c|}{ Frecuencia relativa } \\
\hline Muy bueno & 1 & $1,8 \%$ \\
\hline Bueno & 2 & $3,6 \%$ \\
\hline Regular & 33 & $60,0 \%$ \\
\hline Malo & 19 & $34,5 \%$ \\
\hline
\end{tabular}


Análisis: El 60\% de los clientes considera que la calidad de la lencería (tipo de materiales, textura, suavidad) es regular y no se sienten a gusto con la misma.

\section{Seguridad de las cabañas}

Cuadro 8. Percepción del turista internacional con respecto a la seguridad de las cabañas

\begin{tabular}{|c|r|r|}
\hline Seguridad de las cabañas & Frecuencia absoluta & \multicolumn{1}{|c|}{ Frecuencia relativa } \\
\hline Muy bueno & 8 & $14,5 \%$ \\
\hline Bueno & 3 & $5,5 \%$ \\
\hline Regular & 28 & $50,9 \%$ \\
\hline Malo & 16 & $29,1 \%$ \\
\hline
\end{tabular}

Análisis: El 50,9\% de los turistas considera que la seguridad de las cabañas es regular y que se debería mejorar la misma.

\section{Comodidad de las cabañas}

Cuadro 9. Percepción del turista internacional con respecto a la comodidad de las cabañas

\begin{tabular}{|c|r|r|}
\hline Comodidad de las cabañas & \multicolumn{1}{|c|}{ Frecuencia absoluta } & \multicolumn{1}{c|}{ Frecuencia relativa } \\
\hline Muy bueno & 35 & $63,6 \%$ \\
\hline Bueno & 10 & $18,2 \%$ \\
\hline Regular & 7 & $12,7 \%$ \\
\hline Malo & 3 & $5,5 \%$ \\
\hline
\end{tabular}

Análisis: El 63.6\% de los visitantes considera que la comodidad de las cabañas es muy buena.

\section{Equipamiento de las cabañas}

Cuadro 10. Percepción del turista internacional con respecto al equipamiento de las cabañas

\begin{tabular}{|c|r|r|}
\hline \hline Equipamiento de las cabañas & \multicolumn{1}{|c|}{ Frecuencia absoluta } & Frecuencia relativa \\
\hline Muy bueno & 47 & $85 \%$ \\
\hline Bueno & 9 & $16 \%$ \\
\hline Regular & 4 & $7 \%$ \\
\hline Malo & 2 & $4 \%$ \\
\hline
\end{tabular}

Análisis: El 85\% de los clientes considera que el equipamiento de las cabañas es muy bueno. 


\section{Limpieza de las habitaciones}

Cuadro 11. Percepción del turista internacional con respecto a la limpieza de las cabañas

\begin{tabular}{|c|r|r|}
\hline Limpieza de las habitaciones & Frecuencia absoluta & \multicolumn{1}{|c|}{ Frecuencia relativa } \\
\hline Muy bueno & 4 & $7,3 \%$ \\
\hline Bueno & 1 & $1,8 \%$ \\
\hline Regular & 33 & $60,0 \%$ \\
\hline Malo & 17 & $30,9 \%$ \\
\hline
\end{tabular}

Análisis: El 60\% de los clientes considera que la limpieza de las habitaciones es regular y que se debería realizar de mejor forma.

\section{Forma de atención}

Cuadro 12. Percepción del turista internacional con respecto a la forma de atención

\begin{tabular}{|c|r|r|}
\hline Forma de atención & Frecuencia absoluta & Frecuencia relativa \\
\hline Muy bueno & 50 & $90,9 \%$ \\
\hline Bueno & 3 & $5,5 \%$ \\
\hline Regular & 1 & $1,8 \%$ \\
\hline Malo & 1 & $1,8 \%$ \\
\hline
\end{tabular}

Análisis: El 90,9\% de los clientes considera que la calidad de la atención es muy buena.

\section{Guianza}

\section{Conocimientos del guía}

Cuadro 13. Percepción del turista internacional con respecto al conocimiento del guía

\begin{tabular}{|c|r|r|}
\hline Conocimientos del guía & Frecuencia absoluta & \multicolumn{1}{|c|}{ Frecuencia relativa } \\
\hline Muy bueno & 5 & $9 \%$ \\
\hline Bueno & 3 & $5 \%$ \\
\hline Regular & 35 & $64 \%$ \\
\hline Malo & 12 & $22 \%$ \\
\hline
\end{tabular}

Análisis: El 64\% de los turistas considera que los conocimientos del guía (fauna, flora y cultura) no son los necesarios para ofrecer el servicio de guianza.

\section{Recomendaciones antes y durante las actividades}

Cuadro 14. Percepción del turista internacional con respecto a las recomendaciones del guía

\begin{tabular}{|c|r|r|}
\hline Recomendaciones dadas & Frecuencia absoluta & Frecuencia relativa \\
\hline Muy bueno & 47 & $85 \%$ \\
\hline Bueno & 5 & $9 \%$ \\
\hline Regular & 2 & $4 \%$ \\
\hline Malo & 1 & $2 \%$ \\
\hline
\end{tabular}


Análisis: El $85 \%$ de los visitantes considera que las recomendaciones dadas antes y durante las actividades son muy buenas.

\section{Información impartida por el guía}

Cuadro 15. Percepción del turista internacional con respecto a información impartida del guía

\begin{tabular}{|c|r|r|}
\hline $\begin{array}{c}\text { Información impartida por el } \\
\text { guía }\end{array}$ & Frecuencia absoluta & \multicolumn{1}{|c|}{ Frecuencia relativa } \\
\hline Muy bueno & 3 & $5 \%$ \\
\hline Bueno & 1 & $2 \%$ \\
\hline Regular & 30 & $55 \%$ \\
\hline Malo & 21 & $38 \%$ \\
\hline
\end{tabular}

Análisis: El 55\% de los clientes considera que la información impartida por el guía durante el recorrido es regular y no facilita el entendimiento.

\section{Interpretación del guía durante el recorrido}

Cuadro 16. Percepción del turista internacional con respecto a interpretación del guía

\begin{tabular}{|c|r|r|}
\hline Interpretación del guía & Frecuencia absoluta & \multicolumn{1}{c|}{ Frecuencia relativa } \\
\hline Muy bueno & 1 & $2 \%$ \\
\hline Bueno & 1 & $2 \%$ \\
\hline Regular & 31 & $56 \%$ \\
\hline Malo & 22 & $40 \%$ \\
\hline
\end{tabular}

Análisis: El 56\% de los visitantes considera que la interpretación del guía durante el recorrido se realiza de forma regular y no satisface sus necesidades.

\section{Facilidades turísticas}

Sendero Mira Loma

Señalética de los senderos

Cuadro 17. Percepción del turista internacional con respecto a la señalética del sendero

\begin{tabular}{|c|r|r|}
\hline Señalética del sendero & Frecuencia absoluta & \multicolumn{1}{|c|}{ Frecuencia relativa } \\
\hline Muy bueno & 48 & $87 \%$ \\
\hline Bueno & 4 & $7 \%$ \\
\hline Regular & 2 & $4 \%$ \\
\hline Malo & 1 & $2 \%$ \\
\hline
\end{tabular}

Análisis: El 87\% de los turistas considera que la señalética del sendero es muy buena. 


\section{Información de los medios interpretativos}

Cuadro 18. Percepción del turista con respecto a la información de los medios interpretativos

\begin{tabular}{|c|r|r|}
\hline Medios interpretativos & Frecuencia absoluta & \multicolumn{1}{|c|}{ Frecuencia relativa } \\
\hline Muy bueno & 7 & $13 \%$ \\
\hline Bueno & 4 & $7 \%$ \\
\hline Regular & 25 & $45 \%$ \\
\hline Malo & 19 & $35 \%$ \\
\hline
\end{tabular}

Análisis: El 45\% de los turistas considera que la información de los medios interpretativos del sendero no es buena.

\section{Facilidades del sendero}

Cuadro 19. Percepción del turista internacional con respecto a las facilidades del sendero

\begin{tabular}{|c|r|r|}
\hline Facilidades del sendero & Frecuencia absoluta & \multicolumn{1}{|c|}{ Frecuencia relativa } \\
\hline Muy bueno & 2 & $4 \%$ \\
\hline Bueno & 1 & $2 \%$ \\
\hline Regular & 38 & $69 \%$ \\
\hline Malo & 14 & $25 \%$ \\
\hline
\end{tabular}

Análisis: El 69\% de los turistas considera que las facilidades existentes en el sendero (basureros y puestos de descanso) no son buenas.

\section{Sendero Simón Bolívar}

\section{Señalética del sendero}

Cuadro 20. Percepción del turista internacional con respecto a la señalética del sendero

\begin{tabular}{|c|r|r|}
\hline Señalética del sendero & Frecuencia absoluta & \multicolumn{1}{|c|}{ Frecuencia relativa } \\
\hline Muy bueno & 47 & $85 \%$ \\
\hline Bueno & 5 & $9 \%$ \\
\hline Regular & 2 & $4 \%$ \\
\hline Malo & 1 & $2 \%$ \\
\hline
\end{tabular}

Análisis: El $85 \%$ de los clientes considera que la señalética del sendero es muy buena.

\section{Información de los medios interpretativos}

Cuadro 21. Percepción del turista con respecto a la información de los medios interpretativos

\begin{tabular}{|c|r|r|}
\hline Medios interpretativos & Frecuencia absoluta & \multicolumn{1}{|c|}{ Frecuencia relativa } \\
\hline Muy bueno & 6 & $11 \%$ \\
\hline Bueno & 2 & $4 \%$ \\
\hline Regular & 25 & $45 \%$ \\
\hline Malo & 22 & $40 \%$ \\
\hline
\end{tabular}


Análisis: El 45\% de los turistas considera que la información de los medios interpretativos del sendero es regular y no cumple con sus exigencias y requerimientos.

\section{Facilidades del sendero}

Cuadro 22. Percepción del turista internacional con respecto a las facilidades del sendero

\begin{tabular}{|c|r|r|}
\hline Facilidades del sendero & Frecuencia absoluta & \multicolumn{1}{|c|}{ Frecuencia relativa } \\
\hline Muy bueno & 6 & $11 \%$ \\
\hline Bueno & 5 & $9 \%$ \\
\hline Regular & 25 & $45 \%$ \\
\hline Malo & 19 & $35 \%$ \\
\hline
\end{tabular}

Análisis: El $45 \%$ de los turistas considera que las facilidades existentes en el sendero (basureros y puestos de descanso) no son buenas.

\section{Museo de la Llama}

\section{Interpretación por parte del mediador}

Cuadro 23. Percepción del turista con respecto a la interpretación del mediador

\begin{tabular}{|c|r|r|}
\hline $\begin{array}{c}\text { Interpretación por parte del } \\
\text { mediador }\end{array}$ & Frecuencia absoluta & \multicolumn{1}{|c|}{ Frecuencia relativa } \\
\hline Muy bueno & 2 & $4 \%$ \\
\hline Bueno & 1 & $2 \%$ \\
\hline Regular & 28 & $51 \%$ \\
\hline Malo & 24 & $44 \%$ \\
\hline
\end{tabular}

Análisis: E1 51\% de los clientes considera que la interpretación por parte del mediador del museo no es buena y no es clara y precisa.

\section{Información de los medios interpretativos}

Cuadro 24. Percepción del turista con respecto a la información de los medios interpretativos

\begin{tabular}{|c|r|r|}
\hline Medios interpretativos & \multicolumn{1}{|c|}{ Frecuencia absoluta } & \multicolumn{1}{|c|}{ Frecuencia relativa } \\
\hline Muy bueno & 5 & $9 \%$ \\
\hline Bueno & 2 & $4 \%$ \\
\hline Regular & 26 & $47 \%$ \\
\hline Malo & 22 & $40 \%$ \\
\hline
\end{tabular}

Análisis: El 47\% de los encuestados considera que la información de los medios interpretativos es regular. 


\section{Resumen del nivel de satisfacción de los clientes internacionales}

Cuadro 25. Nivel de satisfacción de los clientes internacionales

\begin{tabular}{|c|c|c|}
\hline \multicolumn{3}{|c|}{ Nivel de satisfacción del cliente internacional } \\
\hline \multicolumn{3}{|c|}{$\begin{array}{c}\text { Servicios } \\
\end{array}$} \\
\hline \multicolumn{3}{|c|}{ Alimentación } \\
\hline Preparación del platillo & Regular & $36 \%$ \\
\hline Calidad de los ingredientes & Regular & $53 \%$ \\
\hline Presentación del platillo & Regular & $53 \%$ \\
\hline Forma de atención & Muy bueno & $82 \%$ \\
\hline Higiene del platillo & Muy bueno & $87 \%$ \\
\hline Tiempo de espera & Regular & $60 \%$ \\
\hline \multicolumn{3}{|c|}{ Hospedaje } \\
\hline Calidad de la lencería & Regular & $60 \%$ \\
\hline Seguridad de las cabañas & Regular & $51 \%$ \\
\hline Comodidad de las cabañas & Muy bueno & $63 \%$ \\
\hline Equipamiento de las cabañas & Muy bueno & $85 \%$ \\
\hline Limpieza de las habitaciones & Regular & $60 \%$ \\
\hline Forma de atención & Muy bueno & $91 \%$ \\
\hline \multicolumn{3}{|c|}{ Guianza } \\
\hline Conocimientos del guía & Regular & $64 \%$ \\
\hline Recomendaciones antes y durante las actividades & Muy bueno & $85 \%$ \\
\hline Información impartida por el guía & Regular & $55 \%$ \\
\hline Interpretación del guía durante el recorrido & Regular & $56 \%$ \\
\hline \multicolumn{3}{|c|}{ Facilidades turísticas } \\
\hline \multicolumn{3}{|c|}{ Sendero Mira Loma } \\
\hline Señalética de los senderos & Muy bueno & $87 \%$ \\
\hline Información de los medios interpretativos & Regular & $45 \%$ \\
\hline Facilidades del sendero & Regular & $69 \%$ \\
\hline \multicolumn{3}{|c|}{ Sendero Simón Bolívar } \\
\hline Señalética de los senderos & Muy bueno & $85 \%$ \\
\hline Información de los medios interpretativos & Regular & $45 \%$ \\
\hline Facilidades del sendero & Regular & $45 \%$ \\
\hline \multicolumn{3}{|c|}{$\begin{array}{r}\text { Museo de la Llama } \\
\end{array}$} \\
\hline Interpretación por parte del mediador & Regular & $51 \%$ \\
\hline Información de los medios interpretativos & Regular & $47 \%$ \\
\hline
\end{tabular}

\section{Nivel de satisfacción del cliente nacional}

\section{Alimentación}

\section{Preparación del platillo}

Cuadro 26. Percepción del turista nacional con respecto a la preparación del platillo

\begin{tabular}{|c|r|r|}
\hline Preparación del platillo & Frecuencia absoluta & Frecuencia relativa \\
\hline Muy bueno & 5 & $15,6 \%$ \\
\hline Bueno & 4 & $12,5 \%$ \\
\hline Regular & 15 & $46,9 \%$ \\
\hline Malo & 8 & $25,0 \%$ \\
\hline
\end{tabular}


Análisis: El 46.9\% de los turistas consideran que la forma de preparación del platillo en cuanto a sabor, olor y color es regular.

\section{Calidad de los ingredientes}

Cuadro 27. Percepción del turista nacional con respecto a la calidad de los ingredientes

\begin{tabular}{|c|r|r|}
\hline Calidad de los ingredientes & Frecuencia absoluta & Frecuencia relativa \\
\hline Muy bueno & 6 & $19 \%$ \\
\hline Bueno & 3 & $9 \%$ \\
\hline Regular & 14 & $44 \%$ \\
\hline Malo & 9 & $28 \%$ \\
\hline
\end{tabular}

Análisis: El 44\% de los clientes considera que la calidad de los ingredientes (frescos, orgánicos, naturales, de temporada) que se utilizan para elaborar los platillos es regular.

\section{Presentación del platillo}

Cuadro 28. Percepción del turista nacional con respecto a la presentación del platillo

\begin{tabular}{|c|r|r|}
\hline Presentación del platillo & Frecuencia absoluta & Frecuencia relativa \\
\hline Muy bueno & 4 & $13 \%$ \\
\hline Bueno & 1 & $3 \%$ \\
\hline Regular & 17 & $53 \%$ \\
\hline Malo & 10 & $31 \%$ \\
\hline
\end{tabular}

Análisis: El 53\% de los visitantes considera que la presentación del platillo (montaje del plato, decoración del plato) es regular.

\section{Forma de atención}

Cuadro 29. Percepción del turista nacional con respecto a la forma de atención

\begin{tabular}{|c|r|r|}
\hline Forma de atención & Frecuencia absoluta & Frecuencia relativa \\
\hline Muy bueno & 29 & $91 \%$ \\
\hline Bueno & 1 & $3 \%$ \\
\hline Regular & 1 & $3 \%$ \\
\hline Malo & 1 & $3 \%$ \\
\hline
\end{tabular}

Análisis: El 91\% de los clientes considera que la forma de atención en el servicio de alimentación es muy buena. 


\section{Higiene del platillo}

Cuadro 30. Percepción del turista nacional con respecto a la higiene del platillo

\begin{tabular}{|c|r|r|}
\hline Higiene del platillo & Frecuencia absoluta & \multicolumn{1}{|c|}{ Frecuencia relativa } \\
\hline Muy bueno & 28 & $88 \%$ \\
\hline Bueno & 1 & $3 \%$ \\
\hline Regular & 2 & $6 \%$ \\
\hline Malo & 1 & $3 \%$ \\
\hline
\end{tabular}

Análisis: E1 88\% de los turistas considera que la higiene de los platillos es muy buena.

\section{Tiempo de espera}

Cuadro 31. Percepción del turista nacional con respecto al tiempo de espera

\begin{tabular}{|c|r|r|}
\hline Tiempo de espera & Frecuencia absoluta & \multicolumn{1}{|c|}{ Frecuencia relativa } \\
\hline Muy bueno & 5 & $16 \%$ \\
\hline Bueno & 2 & $6 \%$ \\
\hline Regular & 17 & $53 \%$ \\
\hline Malo & 8 & $25 \%$ \\
\hline
\end{tabular}

Análisis: El 53\% de los visitantes considera que el tiempo de espera en el servicio de alimentación es regular ya que el mismo no se ofrece de una forma rápida y eficiente.

\section{Hospedaje}

\section{Calidad de la lencería}

Cuadro 32. Percepción del turista nacional con respecto a la calidad de la lencería

\begin{tabular}{|c|r|r|}
\hline Calidad de la lencería & Frecuencia absoluta & \multicolumn{1}{|c|}{ Frecuencia relativa } \\
\hline Muy bueno & 4 & $12,5 \%$ \\
\hline Bueno & 1 & $3,1 \%$ \\
\hline Regular & 18 & $56,3 \%$ \\
\hline Malo & 9 & $28,1 \%$ \\
\hline
\end{tabular}

Análisis: E1 56,3\% de los clientes considera que la calidad de la lencería (tipo de materiales, textura, suavidad) es regular y no se sienten a gusto con la misma.

\section{Seguridad de las cabañas}

Cuadro 33. Percepción del turista nacional con respecto a la seguridad de las cabañas

\begin{tabular}{|c|r|r|}
\hline Seguridad de las cabañas & Frecuencia absoluta & \multicolumn{1}{|c|}{ Frecuencia relativa } \\
\hline Muy bueno & 3 & $9,4 \%$ \\
\hline Bueno & 1 & $3,1 \%$ \\
\hline Regular & 19 & $59,4 \%$ \\
\hline Malo & 9 & $28,1 \%$ \\
\hline
\end{tabular}


Análisis: E1 59,4\% de los turistas considera que la seguridad de las cabañas es regular y que se debería mejorar la misma.

\section{Comodidad de las cabañas}

Cuadro 34. Percepción del turista nacional con respecto a la comodidad de las cabañas

\begin{tabular}{|c|r|r|}
\hline Comodidad de las cabañas & Frecuencia absoluta & \multicolumn{1}{|c|}{ Frecuencia relativa } \\
\hline Muy bueno & 27 & $84,4 \%$ \\
\hline Bueno & 1 & $3,1 \%$ \\
\hline Regular & 2 & $6,3 \%$ \\
\hline Malo & 2 & $6,3 \%$ \\
\hline
\end{tabular}

Análisis: El 84,4\% de los visitantes considera que la comodidad de las cabañas es muy buena.

\section{Equipamiento de las cabañas}

Cuadro 35. Percepción del turista nacional con respecto al equipamiento de las cabañas

\begin{tabular}{|c|r|r|}
\hline Equipamiento de las cabañas & Frecuencia absoluta & Frecuencia relativa \\
\hline Muy bueno & 29 & $91 \%$ \\
\hline Bueno & 1 & $3 \%$ \\
\hline Regular & 1 & $3 \%$ \\
\hline Malo & 1 & $3 \%$ \\
\hline
\end{tabular}

Análisis: El 85\% de los clientes considera que el equipamiento de las cabañas es muy bueno.

\section{Limpieza de las habitaciones}

Cuadro 36. Percepción del turista nacional con respecto a la limpieza de las cabañas

\begin{tabular}{|c|r|r|}
\hline Limpieza de las habitaciones & Frecuencia absoluta & \multicolumn{1}{|c|}{ Frecuencia relativa } \\
\hline Muy bueno & 5 & $15,6 \%$ \\
\hline Bueno & 1 & $3,1 \%$ \\
\hline Regular & 16 & $50,0 \%$ \\
\hline Malo & 10 & $31,3 \%$ \\
\hline
\end{tabular}

Análisis: E1 50\% de los clientes considera que la limpieza de las habitaciones es regular y que se debería realizar de mejor forma.

\section{Forma de atención}

Cuadro 37. Percepción del turista nacional con respecto a la forma de atención

\begin{tabular}{|c|r|r|}
\hline Forma de atención & Frecuencia absoluta & \multicolumn{1}{|c|}{ Frecuencia relativa } \\
\hline Muy bueno & 28 & $87,5 \%$ \\
\hline Bueno & 2 & $6,3 \%$ \\
\hline Regular & 1 & $3,1 \%$ \\
\hline Malo & 1 & $3,1 \%$ \\
\hline
\end{tabular}


Análisis: El 87,5\% de los clientes considera que la calidad de la atención es muy buena.

\section{Guianza}

\section{Conocimientos del guía}

Cuadro 38. Percepción del turista nacional con respecto al conocimiento del guía

\begin{tabular}{|c|r|r|}
\hline Conocimientos del guía & Frecuencia absoluta & Frecuencia relativa \\
\hline Muy bueno & 3 & $9 \%$ \\
\hline Bueno & 1 & $3 \%$ \\
\hline Regular & 19 & $59 \%$ \\
\hline Malo & 9 & $28 \%$ \\
\hline
\end{tabular}

Análisis: El 59\% de los turistas considera que los conocimientos del guía (fauna, flora y cultura) no son los necesarios para ofrecer el servicio de guianza.

\section{Recomendaciones antes y durante las actividades}

Cuadro 39. Percepción del turista nacional con respecto a las recomendaciones del guía

\begin{tabular}{|c|r|r|}
\hline \hline Recomendaciones dadas & Frecuencia absoluta & Frecuencia relativa \\
\hline Muy bueno & 28 & $88 \%$ \\
\hline Bueno & 2 & $6 \%$ \\
\hline Regular & 1 & $3 \%$ \\
\hline Malo & 1 & $3 \%$ \\
\hline
\end{tabular}

Análisis: El $88 \%$ de los visitantes considera que las recomendaciones dadas antes y durante las actividades son muy buenas.

\section{Información impartida por el guía}

Cuadro 40. Percepción del turista nacional con respecto a información impartida del guía

\begin{tabular}{|c|r|r|}
\hline $\begin{array}{c}\text { Información impartida por el } \\
\text { guía }\end{array}$ & Frecuencia absoluta & \multicolumn{1}{c|}{ Frecuencia relativa } \\
\hline Muy bueno & 5 & $16 \%$ \\
\hline Bueno & 3 & $9 \%$ \\
\hline Regular & 18 & $56 \%$ \\
\hline Malo & 6 & $19 \%$ \\
\hline
\end{tabular}

Análisis: El 56\% de los clientes considera que la información impartida por el guía durante el recorrido es regular y no facilita el entendimiento. 


\section{Interpretación del guía durante el recorrido}

Cuadro 41. Percepción del turista nacional con respecto a la interpretación del guía

\begin{tabular}{|c|r|r|}
\hline $\begin{array}{c}\text { Interpretación del guía durante el } \\
\text { recorrido }\end{array}$ & Frecuencia absoluta & Frecuencia relativa \\
\hline Muy bueno & 3 & $9 \%$ \\
\hline Bueno & 2 & $6 \%$ \\
\hline Regular & 19 & $59 \%$ \\
\hline Malo & 8 & $25 \%$ \\
\hline
\end{tabular}

Análisis: El 59\% de los visitantes considera que la interpretación del guía durante el recorrido se realiza de forma regular y no satisface sus necesidades.

\section{Facilidades turísticas}

Sendero Mira Loma

\section{Señalética de los senderos}

Cuadro 42. Percepción del turista nacional con respecto a la señalética del sendero

\begin{tabular}{|c|r|r|}
\hline Señalética del sendero & \multicolumn{1}{|c|}{ Frecuencia absoluta } & \multicolumn{1}{|c|}{ Frecuencia relativa } \\
\hline Muy bueno & 28 & $88 \%$ \\
\hline Bueno & 2 & $6 \%$ \\
\hline Regular & 1 & $3 \%$ \\
\hline Malo & 1 & $2 \%$ \\
\hline
\end{tabular}

Análisis: El 88\% de los turistas considera que la señalética del sendero es muy buena.

\section{Información de los medios interpretativos}

Cuadro 43. Percepción del turista con respecto a la información de los medios interpretativos

\begin{tabular}{|c|r|r|}
\hline Medios interpretativos & Frecuencia absoluta & \multicolumn{1}{|c|}{ Frecuencia relativa } \\
\hline Muy bueno & 4 & $13 \%$ \\
\hline Bueno & 2 & $6 \%$ \\
\hline Regular & 18 & $56 \%$ \\
\hline Malo & 8 & $25 \%$ \\
\hline
\end{tabular}

Análisis: El 56\% de los turistas considera que la información de los medios interpretativos del sendero no es buena. 
Facilidades del sendero

Cuadro 44. Percepción del turista nacional con respecto a las facilidades del sendero

\begin{tabular}{|c|r|r|}
\hline Facilidades del sendero & Frecuencia absoluta & \multicolumn{1}{|c|}{ Frecuencia relativa } \\
\hline Muy bueno & 5 & $16 \%$ \\
\hline Bueno & 1 & $3 \%$ \\
\hline Regular & 19 & $59 \%$ \\
\hline Malo & 9 & $28 \%$ \\
\hline
\end{tabular}

Análisis: $59 \%$ de los turistas considera que las facilidades existentes en el sendero (basureros y puestos de descanso) no son buenas.

\section{Sendero Simón Bolívar}

Señalética del sendero

Cuadro 45. Percepción del turista nacional con respecto a la señalética del sendero

\begin{tabular}{|c|r|r|}
\hline Señalética del sendero & \multicolumn{1}{|c|}{ Frecuencia absoluta } & \multicolumn{1}{c|}{ Frecuencia relativa } \\
\hline Muy bueno & 26 & $81,3 \%$ \\
\hline Bueno & 4 & $12,5 \%$ \\
\hline Regular & 1 & $3,1 \%$ \\
\hline Malo & 1 & $3,1 \%$ \\
\hline
\end{tabular}

Análisis: El 81,3\% de los clientes considera que la señalética del sendero es muy buena.

\section{Información de los medios interpretativos}

Cuadro 46. Percepción del turista nacional con respecto a la información de los medios interpretativos

\begin{tabular}{|c|r|r|}
\hline Medios interpretativos & Frecuencia absoluta & \multicolumn{1}{|c|}{ Frecuencia relativa } \\
\hline Muy bueno & 2 & $6 \%$ \\
\hline Bueno & 2 & $6 \%$ \\
\hline Regular & 19 & $59 \%$ \\
\hline Malo & 9 & $28 \%$ \\
\hline
\end{tabular}

Análisis: E1 59\% de los turistas considera que la información de los medios interpretativos del sendero es regular y no cumple con sus exigencias y requerimientos.

\section{Facilidades del sendero}

Cuadro 47. Percepción del turista nacional con respecto a las facilidades del sendero

\begin{tabular}{|c|r|r|}
\hline Facilidades del sendero & Frecuencia absoluta & \multicolumn{1}{|c|}{ Frecuencia relativa } \\
\hline Muy bueno & 4 & $13 \%$ \\
\hline Bueno & 1 & $3 \%$ \\
\hline Regular & 15 & $47 \%$ \\
\hline Malo & 12 & $38 \%$ \\
\hline
\end{tabular}


Análisis El $47 \%$ de los turistas considera que las facilidades existentes en el sendero (basureros y puestos de descanso) no son buenas.

\section{Museo de la Llama}

\section{Interpretación por parte del mediador}

Cuadro 48. Percepción del turista nacional con respecto a la interpretación del mediador

\begin{tabular}{|c|r|r|}
\hline $\begin{array}{c}\text { Interpretación por parte del } \\
\text { mediador }\end{array}$ & Frecuencia absoluta & \multicolumn{1}{|c|}{ Frecuencia relativa } \\
\hline Muy bueno & 1 & $3 \%$ \\
\hline Bueno & 1 & $3 \%$ \\
\hline Regular & 16 & $50 \%$ \\
\hline Malo & 14 & $44 \%$ \\
\hline
\end{tabular}

Análisis: El 50\% de los clientes considera que la interpretación por parte del mediador del museo no es buena y no es clara y precisa.

\section{Información de los medios interpretativos}

Cuadro 49. Percepción del turista nacional con respecto a la información de los medios interpretativos

\begin{tabular}{|c|r|r|}
\hline Medios interpretativos & \multicolumn{1}{|c|}{ Frecuencia absoluta } & \multicolumn{1}{|c|}{ Frecuencia relativa } \\
\hline Muy bueno & 4 & $12,5 \%$ \\
\hline Bueno & 1 & $3,1 \%$ \\
\hline Regular & 15 & $46,9 \%$ \\
\hline Malo & 12 & $37,5 \%$ \\
\hline
\end{tabular}

Análisis: El 46,9\% de los encuestados considera que la información de los medios interpretativos es regular.

\section{Resumen del nivel de satisfacción de los clientes nacionales}

Cuadro 50. Nivel de satisfacción de los clientes nacionales

\begin{tabular}{|c|c|c|}
\hline \multicolumn{3}{|c|}{ Nivel de satisfacción del cliente nacional } \\
\hline \multicolumn{3}{|c|}{ Servicios } \\
\hline \multicolumn{3}{|c|}{ Alimentación } \\
\hline Preparación del platillo & Regular & $46 \%$ \\
\hline Calidad de los ingredientes & Regular & $44 \%$ \\
\hline Presentación del platillo & Regular & $53 \%$ \\
\hline Forma de atención & Muy bueno & $91 \%$ \\
\hline Higiene del platillo & Muy bueno & $88 \%$ \\
\hline Tiempo de espera & Regular & $53 \%$ \\
\hline \multicolumn{3}{|c|}{ Hospedaje } \\
\hline Calidad de la lencería & Regular & $56 \%$ \\
\hline Seguridad de las cabañas & Regular & $59 \%$ \\
\hline Comodidad de las cabañas & Muy bueno & $84 \%$ \\
\hline Equipamiento de las cabañas & Muy bueno & $91 \%$ \\
\hline
\end{tabular}




\begin{tabular}{|c|c|c|}
\hline Limpieza de las habitaciones & Regular & $50 \%$ \\
\hline Forma de atención & Muy bueno & $87 \%$ \\
\hline \multicolumn{3}{|l|}{ Guianza } \\
\hline Conocimientos del guía & Regular & $59 \%$ \\
\hline Recomendaciones antes y durante las actividades & Muy bueno & $88 \%$ \\
\hline Información impartida por el guía & Regular & $56 \%$ \\
\hline Interpretación del guía durante el recorrido & Regular & $59 \%$ \\
\hline \multicolumn{3}{|l|}{ Facilidades turísticas } \\
\hline \multicolumn{3}{|l|}{ Sendero Mira Loma } \\
\hline Señalética de los senderos & Muy bueno & $88 \%$ \\
\hline Información de los medios interpretativos & Regular & $56 \%$ \\
\hline Facilidades del sendero & Regular & $59 \%$ \\
\hline \multicolumn{3}{|l|}{ Sendero Simón Bolívar } \\
\hline Señalética de los senderos & Muy bueno & $81 \%$ \\
\hline Información de los medios interpretativos & Regular & $59 \%$ \\
\hline Facilidades del sendero & Regular & $47 \%$ \\
\hline \multicolumn{3}{|l|}{$\begin{array}{ll}\text { Museo de la Llama } \\
\end{array}$} \\
\hline Interpretación por parte del mediador & Regular & $50 \%$ \\
\hline Información de los medios interpretativos & Regular & $46 \%$ \\
\hline
\end{tabular}

\section{Propuesta de Mejoramiento del Nivel de Satisfacción del Cliente}

\begin{tabular}{|c|c|}
\hline Servicios & Propuesta de mejoramiento \\
\hline Alimentación & $\begin{array}{l}\text { 1. Capacitar al personal del restaurante con el fin de mejorar } \\
\text { la preparación del platillo en lo que se refiere a sabor y } \\
\text { olor, de esta manera se podrá lograr el máximo nivel de } \\
\text { satisfacción del cliente. } \\
\text { 2. Procurar que los ingredientes que se adquieren para la } \\
\text { preparación de los distintos platillos sean de la mejor } \\
\text { calidad (frescos, orgánicos, naturales, de temporada) con } \\
\text { la finalidad de ofrecer un excelente servicio. } \\
\text { 3. Capacitar al personal en presentación y montaje de platos } \\
\text { con el objetivo de que el platillo sea placentero de } \\
\text { consumir. } \\
\text { 4. Definir los tiempos máximos de repartición de los } \\
\text { diferentes platillos a fin de no causar malestar en los } \\
\text { turistas. }\end{array}$ \\
\hline Hospedaje & $\begin{array}{l}\text { 1. Adquirir lencería de mejor calidad para las camas (buen } \\
\text { material, textura y suavidad) para lograr un excelente } \\
\text { nivel de satisfacción de los visitantes. } \\
\text { 2. Establecer un plan de seguridad para las cabañas con la } \\
\text { finalidad de que las pertenencias del cliente se encuentren } \\
\text { bien salvaguardadas y protegidas. Para lo mismo se } \\
\text { pondrán en marcha los siguientes puntos: } \\
\text { a. Los turistas entregarán sus pertenencias de valor al } \\
\text { encargado del CTC con el fin de evitar pérdidas o robos. } \\
\text { b. Se contratará una persona que se encargará de la } \\
\text { seguridad de las cabañas. }\end{array}$ \\
\hline
\end{tabular}




\begin{tabular}{|c|c|}
\hline & $\begin{array}{l}\text { c. Además, cuando los turistas no se encuentren en las } \\
\text { cabañas, las mismas deben estar siempre cerradas con } \\
\text { llave. De esta manera se evitarán problemas. } \\
\text { d. Finalmente, solo las personas encargadas de la limpieza } \\
\text { de las cabañas podrán ingresar a las mismas cuándo los } \\
\text { visitantes no se encuentren allí. } \\
\text { 3. Establecer un cronograma de limpieza de las habitaciones } \\
\text { con el fin de ofrecer un servicio de buena calidad y no } \\
\text { causar malestar en los clientes por falta de aseo. }\end{array}$ \\
\hline Guianza & $\begin{array}{l}\text { 1. Capacitar al grupo de guías en lo que se refiere a flora, } \\
\text { fauna y cultura con la finalidad de que los conocimientos } \\
\text { que brinden a los turistas sean adecuados. } \\
\text { 2. Capacitar al grupo de guías nativas a fin de que la } \\
\text { información que brinden al visitante tenga pertinencia. En } \\
\text { base a esto se podrá ofrecer un servicio de calidad. } \\
\text { 3. Brindar capacitaciones al personal de guianza en técnicas } \\
\text { de guiar para que la interpretación durante el recorrido sea } \\
\text { excelente. }\end{array}$ \\
\hline Facilidades turísticas & $\begin{array}{l}\text { Propuesta de mejoramiento } \\
\end{array}$ \\
\hline Sendero Mira Loma & $\begin{array}{l}\text { 1. Dotar al sendero de basureros. } \\
\text { 2. Implementar puestos de descanso en el sendero. } \\
\text { 3. Mejorar los medios interpretativos existentes en el } \\
\text { sendero con la finalidad de que faciliten el mejor } \\
\text { entendimiento e interpretación de los turistas. }\end{array}$ \\
\hline Sendero Simón Bolívar & $\begin{array}{l}\text { 1. Implementar puestos de descanso en el sendero. } \\
\text { 2. Implementar basureros en el sendero. } \\
\text { 3. Mejorar los medios interpretativos existentes en el } \\
\text { sendero con la finalidad de que los mismos tengan: } \\
\text { a. Información fácil de leer o visualizar. } \\
\text { b. Información pertinente. } \\
\text { c. Mensaje fácil de comprender o interpretar. } \\
\text { d. Información en inglés, español y kichwa. }\end{array}$ \\
\hline Museo de la Llama & $\begin{array}{l}\text { 1. Mejorar los medios interpretativos en cuanto a: } \\
\text { a. Texto claro y preciso. } \\
\text { b. Información en inglés, español y kichwa. } \\
\text { c. Mensaje pertinente y fácil de interpretar. } \\
\text { 2. Capacitar a los mediadores del museo a fin de que la } \\
\text { información que brinden pueda llegar con claridad al } \\
\text { turista. }\end{array}$ \\
\hline
\end{tabular}

\section{Conclusión}

El análisis de la prestación de servicios ha permitido determinar que el nivel de satisfacción de los clientes nacionales e internacionales no es excelente ya que los resultados arrojados por las encuestas aplicadas nos dicen que sus necesidades y requerimientos no son atendidos de la mejor manera.

Hemos establecido que la propuesta de mejoramiento contribuirá a ofrecer un mejor servicio y de esta manera complacer los gustos y exigencias de los turistas o visitantes que llegan al CTC Sumak Kawsay. 
Los servicios y facilidades turísticas necesitan mejorías para poder lograr el máximo nivel de satisfacción del cliente. Además, si seatienden los requerimientos de los turistas se alcanzará excelencia en la calidad.

Con la propuesta planteada se logrará un excelente nivel de satisfacción por parte del cliente y lograremos cumplir sus exigencias y requerimientos de forma óptima. De esta manera el Centro de Turismo Comunitario Sumak Kawsay podrá encaminarse hacia el desarrollo y mantener altos niveles de calidad.

\section{References:}

1. Cruz Blasco, M. (2012). "Turismo, identidad y reivindicación sociocultural en Chile". Turismo y Sostenibilidad: V Jornadas de Investigación en Turismo, Universidad de Sevilla, Sevilla, Pp. 127147.

2. FEPTCE (2007). Federación Plurinacional de Turismo Comunitario del Ecuador. Manual de calidad para la gestión de turismo comunitario del Ecuador.

3. MCPEYC (2014). "Ministerio Coordinador de Producción, Empleo Y Competitividad".

4. MINTUR (2007). MINTUR (MINISTERIO DE TURISMO DEL ECUADOR). Diseño del Plan Estratégico de Desarrollo de Turismo Sostenible para el Ecuador Plandetur 2020. Mintur Ed. Bid. QuitoEcuador.

5. Prieto, M. (2011). Espacios en disputa: el turismo en Ecuador. FLACSO, Sede Ecuador, 1a edición. Quito - Ecuador.

6. SENPLADES (2013). "Secretaría Nacional de Planificación y Desarrollo - Plan Nacional de Desarrollo / Plan Nacional para el Buen Vivir". 2013-2017 Resolución 2 Registro Oficial Suplemento 78 de 11-sep-2013 Estado. (2013- 2017) Pp. 13

7. Yapu Gutiérrez Fredy (2006). "Turismo Rural, Economía y Desarrollo Local". 